\title{
Nuclear Quantum Effect and Its Temperature Dependence in Liquid Water from Random Phase Approximation via Artificial Neural Network
}

Yi Yao and Yosuke Kanai

\section{Basis Set Convergence of Electronic Structure Calculation}

For the Random Phase Approximation (RPA) calculation, the exact exchange energy (EXX) and the RPA correlation energy were calculated from the Kohn-Sham single-particle wavefunctions based on the PBE GGA XC approximation

$$
E_{c}^{R P A}=\frac{1}{2 \pi} \int_{0}^{\infty} d \omega \operatorname{Tr}\left[\ln \left\{1-\chi_{K S}(i \omega) v\right\}+\chi_{K S}(i \omega) v\right]
$$

where the non-interacting (Kohn-Sham) response function is given in terms of the Kohn-Sham eigenstates as

$$
\chi_{K S}\left(r_{1}, r_{2}, i \omega\right)=2 \sum_{i}^{o c c} \sum_{a}^{v i r} \frac{\psi_{a}^{*}\left(r_{1}\right) \psi_{i}\left(r_{1}\right) \psi_{a}\left(r_{2}\right) \psi_{i}^{*}\left(r_{2}\right)}{\left(\varepsilon_{a}-\varepsilon_{i}\right)-i \omega}
$$

and $v\left(r_{1}, r_{2}\right)$ is the bare Coulomb interaction. We use the CP2 $\mathrm{K}$ code ${ }^{1-2}$ to perform the RPA calculations within the mixed Gaussian and Planewaves (GPW) formalism ${ }^{1,3-5}$. The so-called direct-RPA approach ${ }^{6}$ is implemented in the CP2K code. The TZVP Gaussian basis set was used $^{7-8}$ together with the planewave cutoff of 800 Ryd for the electron density, and GoedeckerTeter-Hutter (GTH) type pseudopotential was used ${ }^{9}$. Resolution of identity was used for reducing the computational cost of the four center integrals ${ }^{4}$. Because it is presently not feasible to perform RPA-based FPMD simulation with the planewave basis set, SCAN meta-GGA XC was used for assessing the convergence of the TZVP Gaussian basis set. The TZVP basis set result was compared with the converged planewave basis set result with the kinetic energy cutoff of 400 Ryd for the Kohn-Sham wavefunctions, using the CPMD code ${ }^{10}$, to which we added SCAN meta-GGA XC approximation ${ }^{11}$. Figure S1 shows that the oxygen-oxygen radial distribution function (RDF) at $300 \mathrm{~K}$ and the temperature-dependent diffusion coefficients using 
these two basis sets. As can be seen, differences are insignificant, especially when one considers the statistical errors. The GPW (TZVP/800Ryd)-based FPMD is well converged with respect to the planewave FPMD simulation performed at the converged planewave kinetic energy cutoff of 400 Ryd.
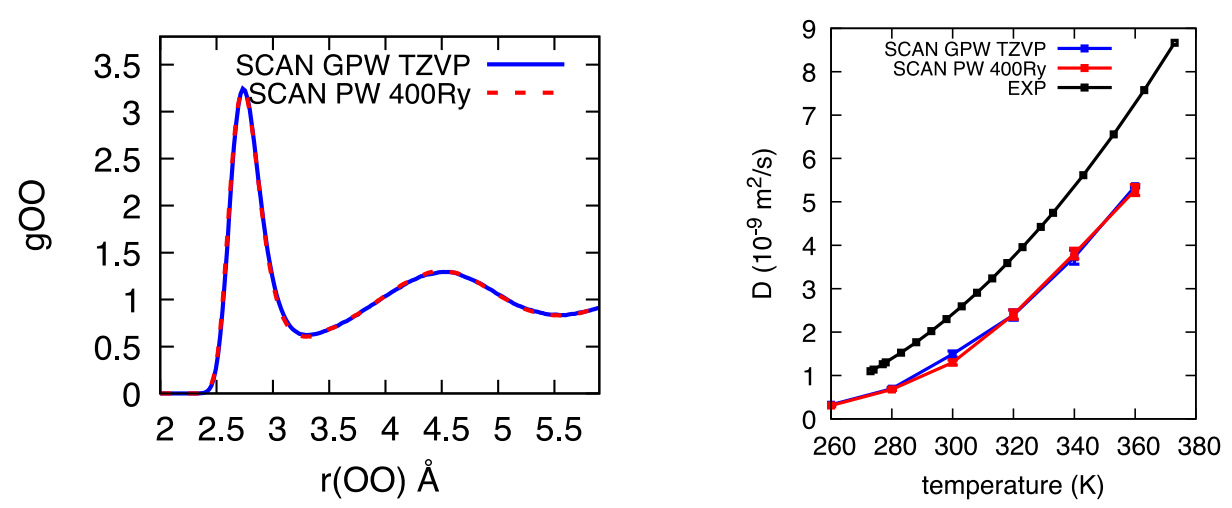

Figure S1. Comparison of FPMD simulations based on GPW (TZVP/800Ryd) and plane-waves (400 Ryd for KS wavefunctions) at the SCAN meta-GGA XC level of theory. (Left) The oxygen-oxygen radial distribution function of liquid water at $300 \mathrm{~K}$. (Right) The temperaturedependent diffusion coefficients.

\section{Neutral Network Model}

The artificial neural network (NN) model was developed following our earlier work on the NN model for SCAN meta-GGA XC functional ${ }^{12}$ in the exactly the same manner, and only total energies are included in the loss function. We used the AENET code to train the artificial NN model. The Behler's method was used with 51 symmetry functions for hydrogen atoms and 46 symmetry functions for oxygen atoms as discussed by Behler and Parrinello ${ }^{13-14}$. Two hidden layers are used for the $\mathrm{NN}$ model with 25 nodes in each layer. The training structure set contains 10449 structures in total. They are a combination of structures directly from the FPMD simulation (in the Car-Parrinello extended Lagrangian formalism) based SCAN meta-GGA and TRPMD simulation based on the pre-trained NN-SCAN model. For each evenly separated temperature ranging from $260 \mathrm{~K}$ to $400 \mathrm{~K}, 8$ independent FPMD trajectories are run for $30 \mathrm{ps}$ using a 55-atom cubic simulation cell. After a 5ps equilibration period, 130 snapshots are evenly chosen from each trajectory. This gives 8320 training structures. Additional 2129 structures are taken from TRPMD simulation using the NN-SCAN model trained based on these 8320 structures. We have shown that this training set is large enough to adequately capture liquid water structural and dynamics properties in our earlier work with SCAN meta-GGA XC functional. $90 \%$ of the structures in the set are randomly selected for training the NN model for the RPA+EXX calculation, and $10 \%$ of the structures are used for testing. avoid overfitting, we adopt the early stopping method ${ }^{15}$. The root mean square error was converged to $<0.5 \mathrm{meV} /$ atom. The oxygen-oxygen RDF is available from the first-principles Monte Carlo NpT simulation by Del Ben, et. al. ${ }^{16}$, and an excellent agreement was obtained for our NN-RPA model as shown in 
Figure S2. Note that the NN force field we developed here is strictly for liquid water system since as the training data includes only liquid water.

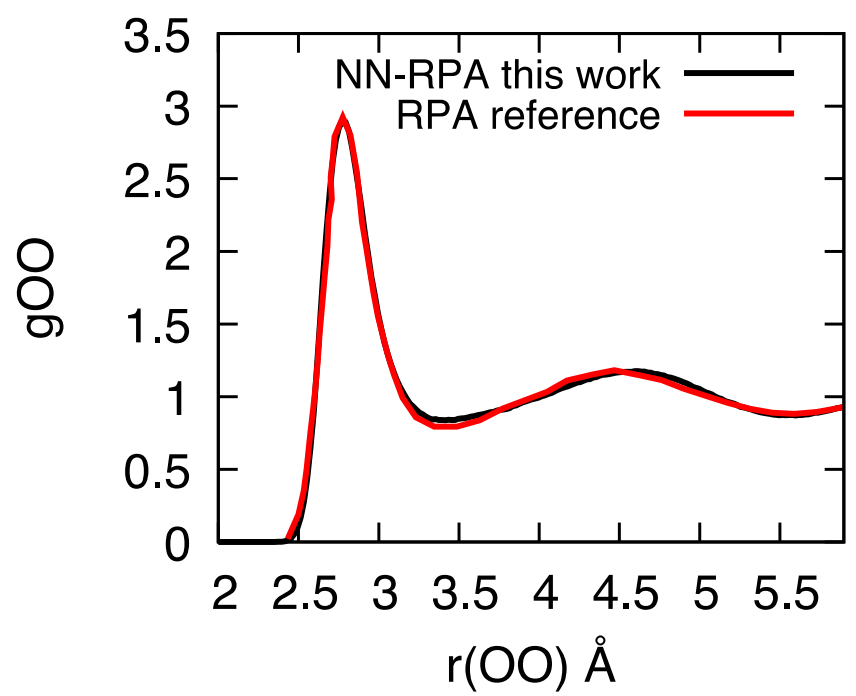

Figure S2. Comparison between our NN-RPA model (NN-RPA this work) and the firstprinciples Monte Carlo simulation of NpT ensemble (RPA reference) ${ }^{16}$ for the oxygen-oxygen radial distribution function, $\mathrm{gOO}$, at room temperature. We used a cubic simulation cell of $11.8172 \AA$ with the periodic boundary conditions with $55 \mathrm{H}_{2} \mathrm{O}$ molecules at $300 \mathrm{~K}$, corresponding to the water density of $0.997 \mathrm{~g} / \mathrm{cm}^{3}$. The first-principles Monte Carlo NpT simulation (with 26800 cycles) used $64 \mathrm{H}_{2} \mathrm{O}$ molecules in a cubic simulation cell, and the simulation yielded the average density of $0.994 \mathrm{~g} / \mathrm{cm}^{3}$ at $295 \mathrm{~K}$. The statistical error bars are smaller than the thickness of the line.

Figure S3 shows the scatter plots for the potential energies calculated by direct first principles calculation and NN model for the EXX+RPA (left) and SCAN meta-GGA XC (right) levels of theory. The results for the SCAN meta-GGA XC are taken from our previous work ${ }^{12}$. The NN models for the RPA+EXX and SCAN meta-GGA give the model accuracies that are essentially the same in terms of the potential energy (i.e. $<0.5 \mathrm{meV} / \mathrm{atom}$ ). These results indicate that the RPA+EXX NN model is as representative of the RPA+EXX theory as the NN model was for the SCAN meta-GGA work ${ }^{12}$. For the SCAN meta-GGA XC, we have provided more extensive comparison between the $\mathrm{NN}$ model and the underlying electronic structure calculation for various properties including the difficult case of the diffusion coefficient because the forces are readily available for the SCAN meta-GGA XC level of theory for the direct FPMD simulation. 

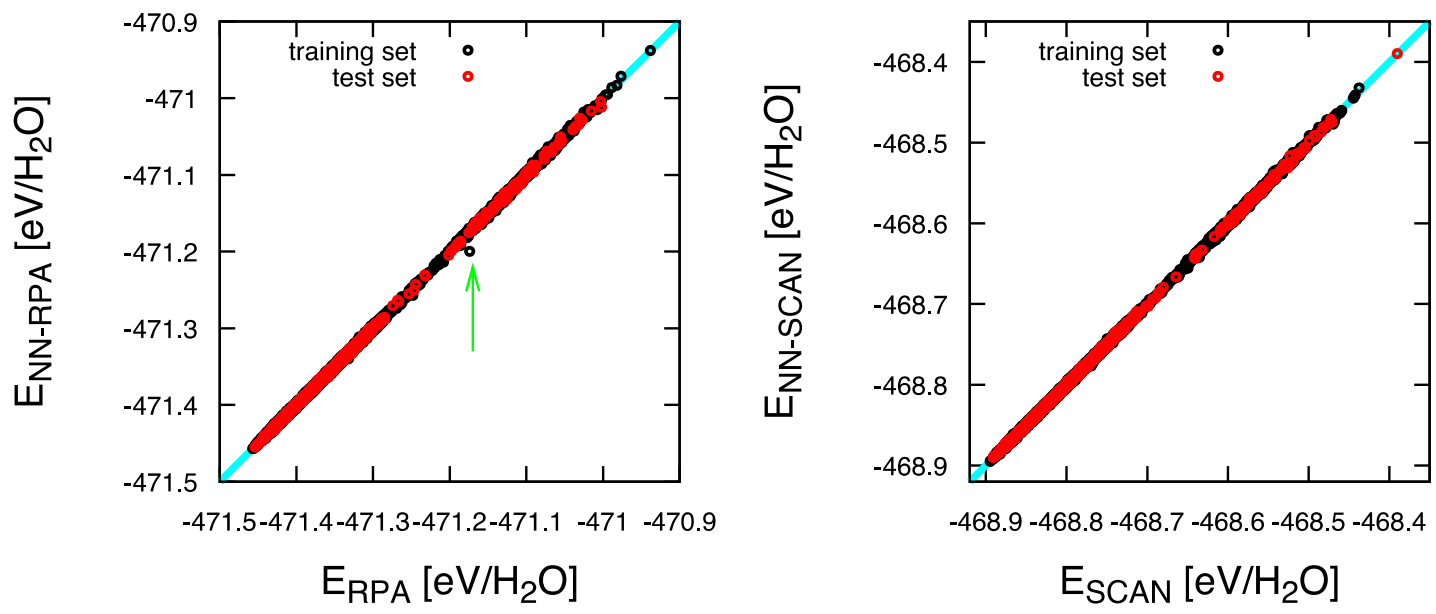

Figure S3. The scatter plots for the potential energies calculated by direct first principles calculation and NN model for the EXX+RPA (left) and SCAN meta-GGA XC (right) levels of theory. The plots include total of 10449 configurations, including the training and test sets. The diagonal line indicates perfect agreement. The green arrow on the left plot indicates slightly outside the agreement due to an extremely rare configuration of self-ionization, which occurred in the training set by chance. Such a configuration does not exist in our MD simulations presented in the main text.

\section{$\underline{\text { Temperature Dependence of Liquid Density }}$}

For simulating liquid water in the temperature range of $260 \mathrm{~K} \sim 360 \mathrm{~K}$, the liquid density of $0.997 \mathrm{~g} / \mathrm{cm}^{2}$, which corresponds to the experimental density at $300 \mathrm{~K}$, was used for convenience. For the lower temperatures, the experimental liquid density remains essentially the same as that of $300 \mathrm{~K}$. The largest deviation in the liquid density occurs for the highest temperature simulated here, $360 \mathrm{~K}\left(0.967 \mathrm{~g} / \mathrm{cm}^{2}\right)$. Still, the change is only $-3 \%$. Figure S4 validates that calculated properties, even rather sensitive quantities like the diffusion coefficient, do not change even if the experimental density is used at $360 \mathrm{~K}$. The calculated diffusion coefficients at $360 \mathrm{~K}$ using the experimental water densities of $300 \mathrm{~K}$ and of $360 \mathrm{~K}$ agree well within the statistical error bars. 


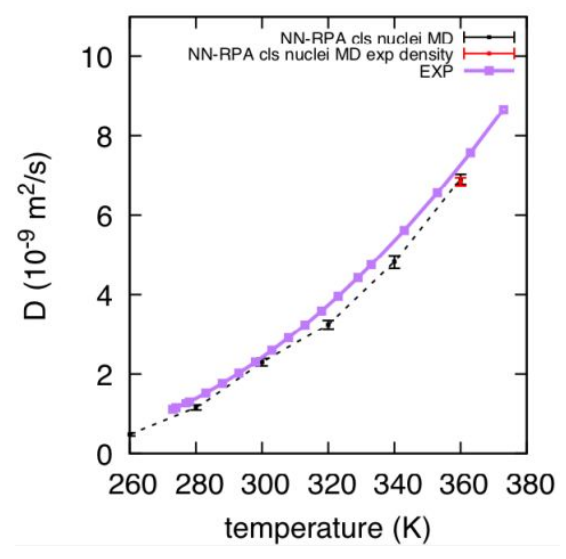

Figure S4. The diffusion coefficient of liquid water calculated by the $\mathrm{NN}$ model for the EXX+RPA with classical nuclei MD with the experimental density at $360 \mathrm{~K}\left(0.967 \mathrm{~g} / \mathrm{cm}^{2}\right)$. The result with the liquid water density of $0.997 \mathrm{~g} / \mathrm{cm}^{2}$, which corresponds to the experimental density at $300 \mathrm{~K}$, and the experimental diffusion coefficients over the temperature range of $260 \sim 360 \mathrm{~K}$ are also shown for comparison.

\section{References}

1. Hutter, J.; Iannuzzi, M.; Schiffmann, F.; VandeVondele, J., cp2k: atomistic simulations of condensed matter systems. Wiley Interdisciplinary Reviews: Computational Molecular Science 2014, 4(1), 15-25.

2. Kühne, T. D.; Iannuzzi, M.; Del Ben, M.; Rybkin, V. V.; Seewald, P.; Stein, F.; Laino, T.; Khaliullin, R. Z.; Schütt, O.; Schiffmann, F.; Golze, D.; Wilhelm, J.; Chulkov, S.; BaniHashemian, M. H.; Weber, V.; Borštnik, U.; Taillefumier, M.; Jakobovits, A. S.; Lazzaro, A.; Pabst, H.; Müller, T.; Schade, R.; Guidon, M.; Andermatt, S.; Holmberg, N.; Schenter, G. K.; Hehn, A.; Bussy, A.; Belleflamme, F.; Tabacchi, G.; Glöß, A.; Lass, M.; Bethune, I.; Mundy, C. J.; Plessl, C.; Watkins, M.; VandeVondele, J.; Krack, M.; Hutter, J., CP2K: An electronic structure and molecular dynamics software package - Quickstep: Efficient and accurate electronic structure calculations. The Journal of Chemical Physics 2020, 152 (19), 194103. 
3. VandeVondele, J.; Krack, M.; Mohamed, F.; Parrinello, M.; Chassaing, T.; Hutter, J., Quickstep: Fast and accurate density functional calculations using a mixed Gaussian and plane waves approach. Computer Physics Communications 2005, 167(2), 103-128.

4. Del Ben, M.; Schütt, O.; Wentz, T.; Messmer, P.; Hutter, J.; VandeVondele, J., Enabling simulation at the fifth rung of DFT: Large scale RPA calculations with excellent time to solution. Computer Physics Communications 2015, 187, 120-129.

5. Del Ben, M.; Hutter, J. r.; VandeVondele, J., Electron correlation in the condensed phase from a resolution of identity approach based on the Gaussian and plane waves scheme. Journal of chemical theory and computation 2013, $9(6), 2654-2671$.

6. Eshuis, H.; Yarkony, J.; Furche, F., Fast computation of molecular random phase approximation correlation energies using resolution of the identity and imaginary frequency integration. The Journal of Chemical Physics 2010, 132 (23), 234114.

7. Dunning Jr, T. H., Gaussian basis sets for use in correlated molecular calculations. I. The atoms boron through neon and hydrogen. The Journal of chemical physics 1989, 90 (2), 10071023.

8. Del Ben, M.; Hutter, J. r.; VandeVondele, J., Second-order Møller-Plesset perturbation theory in the condensed phase: An efficient and massively parallel Gaussian and plane waves approach. Journal of chemical theory and computation 2012, 8(11), 4177-4188.

9. Goedecker, S.; Teter, M.; Hutter, J., Separable dual-space Gaussian pseudopotentials. Physical Review B 1996, 54 (3), 1703.

10. Hutter, J.; Iannuzzi, M., CPMD: Car-Parrinello molecular dynamics. Zeitschrift für Kristallographie - Crystalline Materials 2005, 220 (5-6), 549-551.

11. Yao, Y.; Kanai, Y., Plane-wave pseudopotential implementation and performance of SCAN meta-GGA exchange-correlation functional for extended systems. The Journal of Chemical Physics 2017, 146 (22), 224105.

12. Yao, Y.; Kanai, Y., Temperature dependence of nuclear quantum effects on liquid water via artificial neural network model based on SCAN meta-GGA functional. The Journal of Chemical Physics 2020, 153 (4), 044114.

13. Behler, J.; Parrinello, M., Generalized Neural-Network Representation of HighDimensional Potential-Energy Surfaces. Physical Review Letters 2007, 98 (14), 146401.

14. Behler, J., Atom-centered symmetry functions for constructing high-dimensional neural network potentials. The Journal of Chemical Physics 2011, 134 (7), 074106. 
15. Caruana, R.; Lawrence, S.; Giles, L., Overfitting in neural nets: Backpropagation, conjugate gradient, and early stopping. Advances in neural information processing systems 2001, 402-408.

16. Del Ben, M.; Hutter, J.; VandeVondele, J., Probing the structural and dynamical properties of liquid water with models including non-local electron correlation. The Journal of chemical physics 2015, $143(5), 054506$. 